\title{
Design and Implementation of Engineering Cost Management System
}

\author{
Chen Hai \\ Restoration Project Management Section, General Logistic Management Office, Jinan University, \\ Guangzhou 510000, China
}

Keywords: Cost management; Project management; MVC framework

\begin{abstract}
Large-scale construction projects require a large amount of manpower, material resources and funds, and strict and detailed construction plans and cost analysis are needed in the construction process. However, the traditional project cost budget mainly relies on the construction unit group budget analyst to complete by off-line way. Such kind of project budgeting method has many problems, such as heavy workload, poor reusability, low transparency and poor real-time performance. With the development of computer network technology, People's Daily working mode is changing from offline to online, and work place is changing from office to virtual network platform. Under the background of information age, how to transform the traditional project cost prediction and analysis from the offline work of team members to the online work of professional teams is the common problem faced by all construction units. Based on the above problems, this paper designs and realizes the engineering cost management information system.
\end{abstract}

\section{The theoretical basis and main techniques of the system}

\subsection{Concept of construction engineering cost system}

The system of construction engineering includes the necessary budget and the corresponding comprehensive cost in each stage of construction. In terms of a certain level, the definition of the scope of construction engineering varies from time to time. Generally speaking, it has two main aspects:

(1) From the business level of project cost, project cost is actually integrated whole, management from project start to the end of the final project implementation, all of the cost of the project schedule of human and other related resources integrated management and application, the application requires investors correct treatment project of different stages and reasonable plan and design project of the whole plan. [1]

(2) From the perspective of market, it means that from the beginning of implementation of the whole project, we must make effective use of various resources of the project, which are related to market value and comprehensive information in the market. Only by managing such comprehensive information can the whole project be completed in a better and more economical coordination with yours. In addition, we adopt scientific project tender and bid, this is a kind of effective method to save the project cost, in the process of the implementation of management is to control the engineering cost is an important link of all our project control and budget must send children project subject to tender according to the engineering plan have to but the implementation of strict, ensure the reasonable and unity of the management.

\subsection{Development of construction project cost management system}

(1) The bidding stage of the project. Used to manage the overall system of an engineering important stage. This stage includes the control and implementation of the whole project.

(2) Construction stage of the project. [2] The main work is to complete the corresponding project goal control and plan, strict implementation cost project content principle and related content. At the time of project settlement and construction cost, detailed work project list, as well as project content, shall be quoted and priced around construction.

(3) Settlement stage of shuttle engineering project. The emphasis is to verify the rationality of the 
project and supervise the whole project. This part is mainly to complete relevant bidding tips and provide cost management according to this part.

\subsection{Introduction of the main software environment}

(1) The operating system of the server

This system adopts the current, mainstream appliances in the market, the technology of ASP.NET, and the technology of SQL Server. In addition, we should choose the mainstream systems of Microsoft, such as WIN7 and Win Server.

(2) Client software environment

The main software environment of the system includes operating system, development tools. The operating system is the mainstream operating system of Microsoft, which can be Windows 7 or Windows Sever series, etc. [3] After adopting the three-layer structure, our database will choose SQLServer2005 for development.

\subsubsection{ASP.NET}

Is introduced as the Java programming application scenarios in the network environment, Microsoft in order to secure the advantages in the software industry is decisive, launching a strategic influence of the web development language - ASP.NET, it is not only a language represents a new generation of Microsoft framework, it is a framework based on translation and mature tools, it is compatible with Microsoft some mainstream platform, and his application can be used in the Microsoft platform, relative to other company's software product, its biggest advantage lies in its mature good compatibility. NET platform. In this way, its code and related documentation can have good high coupling and reuse performance. In addition, he can be compatible with mainstream compilation such as WYSIWYG HTML. This integration of the required functional modules into the development framework makes it much easier for software developers to develop, and is favored by a wide range of companies.

\subsubsection{VC development framework}

The MVC pattern is a model-view-controller combination. Is the purpose of the MVC pattern in order to guarantee the security of database and operation between users, it uses an intermediary as a bridge in the interaction of the user and the database, the model provides users with multiple view model, so as to let the user directly operation with data that reached the purpose of separation and high separation, practical benefits at the same time it also improves the efficiency of the whole system, the developer can more effectively to focus on high-level development rather than at the bottom of the details. [4]

(1) Model. This is the body of our entire structure, and it will provide some multipurpose USES for the user to choose, so that the user can operate on the model of the framework without having to design the underlying considerations. This improves system reusability.

(2) view. The view is an interactive interface between the user and the computer. This part requires that we can directly check the view module of the model and update the corresponding functional module.

(3) controller. The controller is the requirement interface for the user to complete a certain function according to the user's input prompt. After the Web sends the request, it reaches the controller layer, and the controller calls the request according to the corresponding module to complete the specific function.

\subsubsection{B/S development framework}

This structure mainly means that the entire $\mathrm{B} / \mathrm{S}$ architecture is divided into three parts: application side processing, logical side processing of the second layer, and server-side processing. The use of the client on behalf of the user level represents the user's processing of the data, the logical part is the intermediate layer's parsing of the user's intention, which is then converted into the database language, and finally the data is transmitted to the server, where the database management system provides the data processing according to the command. "Three-layer 
architecture". These three layers are relatively independent from each other. By switching and debugging the other two layers through the middle layer of web server, the whole program is logically divided into three layers, enabling developers from different departments to develop their modules independently.

\section{Design and analysis of construction cost system}

\subsection{Demand analysis}

The engineering cost information system includes the following parts: audit leader, template designer, template approver, project cost controller, project manager, system manager and supplier member.

Leadership review

This type of user belongs to the management level of the system, which is mainly to check and supervise the whole project, so as to know the progress of the whole project in real time, and download and print the report of the project information.

Project template designer

The main work of this personnel is to design new cost simulation board according to the changes in the market and customer demands, so that the template can be provided for the cost staff to use and be able to carry out engineering management.

Project template approval personnel

The main work of the template approval personnel is to check the template design, review the data if it meets the requirements, and upload the system to the cost engineer for use. The unqualified will return the template to the designer for modification.

Project cost personnel

The cost engineering is the main work of our system. [5] According to the information uploaded by the template, the cost engineer selects the correct template to carry out the cost. It is the activity subject of the whole system.

Project management personnel

Project management is the macro level of the whole system, and the project is the project of the whole cost project. It can manage different construction stages, or the macro allocation of the whole project.

System management personnel

System management is the highest authority of this system. Instead of focusing on engineering projects, they only focus on the management of this cost system itself. They have the authority to modify the information and data of any user.

Market suppliers

The supplier is the supplier of the whole system materials, whose main task is to complete the estimation of the engineering materials and other hardware facilities for users to choose.

\subsection{System design overview}

For the overall design, we need to put forward some system requirements after the realization of the whole system. The following points help us analyze the work requirements of the whole system:

(1) It is in the environment of network resource sharing, and it must guarantee a multi-functional system with comprehensive performance of resource sharing information among various departments;

(2) This system shall realize the display of the whole business and centralized processing of data. The advantage of such centralized processing is that the information resources of the whole system can be Shared and interactive.

(3) The whole project must be managed under control, that is to say, the whole project must have certain constraints and systematic management rules, so that the system can achieve the goal we want;

(4) In addition, we should consider from the following aspects: 
The project of the entire construction cost project should be carried out according to the level, which is beneficial to our management and can manage the whole project reasonably and effectively.

Besides, we should consider the comprehensiveness of the whole project, including the selection rules of cost management and materials, as well as the assistance of various departments.

We need to have clear lists and written instructions for projects so that we can streamline the project.

However, we can accurately calculate the cost of each template, so that we can strictly control the price and cost of the whole project, and the data can serve the whole project well.

(5) the last for the whole project, we must have macro control, such as the whole project between various departments to assist and macro regulation and so on, comprehensive management of complex information must be in the system in the form of clear: system user management subsystem, cost engineering management subsystem, project progress management information subsystem, material equipment management subsystem, query statistics management subsystem.

\section{Detailed design of construction cost system}

Based on the analysis of the first three chapters, relevant functions and technical implementation of each department in the construction project cost management system are explained. In this chapter, we will make detailed functional descriptions of all relevant information.

The functional module diagram of the template management subsystem is shown below.

(1) User management subsystem

The user management subsystem is mainly a module that uses the information of administrative staff, including the functions of adding, deleting, checking and changing. [6] Management work for users of the entire system. After the user enters the system, the user first needs to log in, which is mainly used to receive the user name and password entered by the user. This part is mainly about the user's authentication function, and the user administrator can audit and manage all the data for this part.

(2) Cost management subsystem

The cost management subsystem has the functions of progress review, construction progress supervision, project cost audit, project management, project information query, material plan approval and cost error analysis. We can look up all kinds of funds to be approved. For approved funds, we can also search for corresponding information. After the information is inquired, we will evaluate some of the funds. The estimated information is being audited. If the audit passes, it will be uploaded to the information database. If it fails, the data will be re-examined or submitted to the relevant department for modification.

(3) Engineering information management subsystem

Engineering information management is the whole project of the subsidiary, the information of the project including project component name, as well as engineering materials used in human cost, total engineering and other relevant information we can be divided into several sub project, each worker assigned a few heads in the project management, each responsible for each project will upload the information of the project, in the center database integrate themselves after the completion of the work for the whole project, and finally summarize the whole project to complete.

(4) Material equipment management subsystem

The management subsystem of material equipment is mainly about the management of materials, including compilation, the registration of bidding and entry, etc.

(5) System auxiliary function subsystem

It mainly includes initial parameter setting, data backup, system maintenance and user login. The auxiliary function is mainly the system use function of partial auxiliary information to complete the corresponding function of partial auxiliary system and the user to make better use of the system. 


\section{Conclusion}

Since the 21 st century, more and more construction projects in various regions of China, the construction scale is getting larger and larger. In the past ten years, new and old buildings have been replaced and high-rise buildings have been built up in various provinces and cities, and the rapid development of architectural engineering technology has brought great changes to people's lives. Based on the actual management method and mode used by the engineering company, this paper designs a three-layer architecture design based on the MVC model, and implements the engineering cost information management system according to the actual application requirements of the current engineering cost field.

\section{References}

[1] Wang Xinzhi. Design of highway engineering cost information management system [J]. Transportation, 2015 (02): 01, 114-117.

[2] Sun Zhengmao, Yuying. Design and implementation of construction engineering cost control system [J]. BBS, industry and technology, 2013 (11): 09:60-61.

[3] Wang Yanping. System design of information construction of highway construction cost management [J]. Highway traffic technology (technology), 2012, (06): 263-266.

[4] $\mathrm{Xu}$ Leilei. Research on the design and implementation of construction engineering cost information management system [J]. Information and communications, 2015, (02):117-118.

[5] Gong Ningping. Analysis and design of engineering cost information system based on component [J]. Development and application of computer, 2008, (4):64-66.

[6] Tang Yujiao. Design and implementation of integrated information system of engineering cost [J]. Journal of Guanxi academy of sciences, 2004, (08): 165-167. 